\title{
Wear and friction dependence on grain size
}

\author{
Vladimir Stolyarov ${ }^{1 *}$ \\ ${ }^{1}$ Mechanical Engineering Research Institute of Russian Academy of Sciences, 101990, Moscow, \\ Russia
}

\begin{abstract}
The tribological characteristics of pure metals and ceramics with graphene obtained by electrochemical, deformation and powder metallurgical methods are compared for a coarse-grained and nanocrystalline state. Reducing the grain size to the nanoscale region in the structure helps to reduce wear and friction coefficient compared to conventional analogues. The possibilities of modelling the contact of solids with the atomic structure of the surface are considered.
\end{abstract}

\section{Introduction}

Nanocrystalline (NC) materials, as a result of a significant decrease in grain size and a substantial increase in the volume fraction of their boundaries, exhibit unusual mechanical, physical, and electrochemical properties compared to ordinary polycrystals [1]. High strength and hardness of NC materials is the basis for promising structural applications and therefore stimulates the development and research of new metals, alloys, and compounds. There is a significant number of works devoted to studies of the mechanical behaviour of $\mathrm{NC}$ materials in standard tests for hardness, compression, or tension [2, 3]. However, the mechanisms of friction and wear in NC materials are poorly studied, possibly due to the difficulty of creating massive samples sufficient to study friction and wear. Most of the studies on the wear of the surface layer or coatings were carried out in metal alloys, which showed increased wear resistance of $\mathrm{NC}$ materials in comparison with their coarse-grained (CG) analogues [4]. At the same time, systematic studies of wear in model pure NC metals nanocomposites, in which the effect is not associated with phase transformations, are of great value. Until recently, they were few in number due to difficulties in the synthesis of macroscopic samples suitable for testing. The own and published data for pure NC metals ceramics (copper [5], nickel [6], titanium [7], A12O3/graphene) obtained by electrodeposition, severe plastic deformation and powder metallurgy methods are presented below.

\section{Materials and research methods}

In pure copper, the NC state was obtained by electrodeposition of the initial CG (50-100 $\mu \mathrm{m})$ copper with a purity of $99.99 \%$. The average crystal size in NC copper was $20 \mathrm{~nm}$. Both states were tested for wear under dry friction conditions at room temperature according to the "ball-disk" scheme, in which a WC - Co alloy ball with a diameter of 10

\footnotetext{
* Corresponding author: vlstol@mail.ru
} 
mm slid over a copper disk of $\varnothing 7 \times 2 \mathrm{~mm}$ with an amplitude load of 5 to $40 \mathrm{~N}$ frequencies of $5 \mathrm{~Hz}$.

NC nickel was also obtained by electrodeposition; the processing method is described in detail in [6]. The crystallite size in NC nickel was estimated by the X-ray method and ranged from 8 to $28 \mathrm{~nm}$; the average crystal size was $20 \mathrm{~nm}$. Tribological tests were performed without lubrication according to the "steel ball-disk" scheme, on surfaces cleaned by ultrasound, at a load of $1 \mathrm{~N}$, a frequency of $8 \mathrm{~Hz}$, tangential displacement amplitude of $100 \mu \mathrm{m}$ and a number of cycles of 10000. CG nickel used as a reference element was obtained cold rolling and annealing to form a structure with an average grain size of $61 \mu \mathrm{m}$. Microhardness was measured at a load of $1 \mathrm{~N}$, so that the penetration depth was less than $10 \%$ of the thickness of the deposited layer.

For the study, commercial pure titanium was selected in the form of a hot-rolled bar of $\varnothing 50 \mathrm{~mm}$ containing impurities $0.07 \% \mathrm{C}, 0.18 \% \mathrm{Fe}, 0.10 \% \mathrm{Si}, 0.12 \% \mathrm{O}, 0.04 \% \mathrm{~N}$ and $0.01 \% \mathrm{H}$. The average grain size was $15 \mu \mathrm{m}$. After turning to $\varnothing 40 \mathrm{~mm}$, a bar $100 \mathrm{~mm}$ long was subjected to warm equal channel angular pressing (ECAP), the process of which was described in detail in [7]. Part of the ECAP samples was deformed by cold rolling (CR) by $75 \%$ in order to further refine the structure. As a result, an ultrafine-grained (UFG) microstructure was obtained with a grain size of $0.3 \mu \mathrm{m}$ (after ECAP) and NC microstructure of $0.1 \mu \mathrm{m}$ (after ECAP + CR). A typical NC microstructure in titanium after ECAP and cold rolling is shown in Fig. 1.

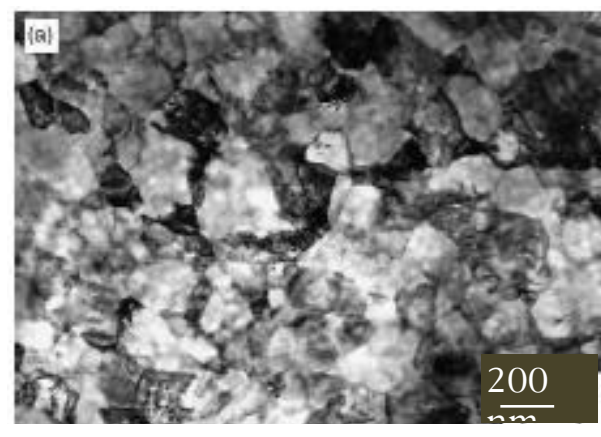

a

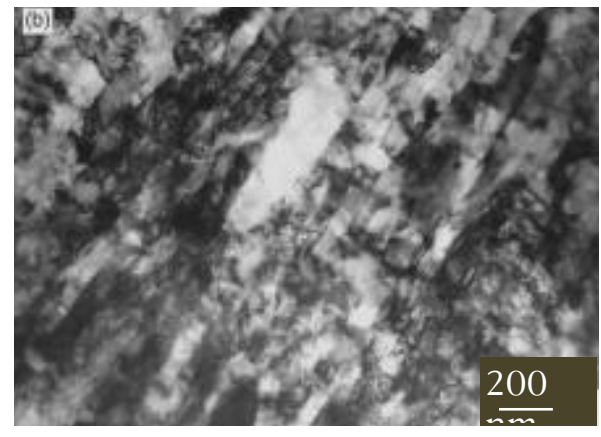

b

Fig. 1. Microstructures of NC titanium in the transverse (a) and longitudinal (b) section.

It is known [8] that the tendency to set and stick (typical for titanium) depends on the resistance of the material to adhesion under shear stresses, which can be estimated by the ratio of tangent $\tau_{\mathrm{nn}}$ and normal stresses $\mathrm{P}_{\mathrm{rn}}$ at the contact of solids. This ratio $\left(\mu_{\mathrm{a}}=\tau_{\mathrm{nn}} / \mathrm{P}_{\mathrm{rn}}\right)$ is known as the adhesive component of the friction coefficient which the smaller, the smaller setting and sticking. The measurement of $\mu_{\mathrm{a}}$ was carried out according to the method of [9] on a setup simulating friction conditions between a hemispherical indenter made of WCo alloy $(\varnothing 2.5 \times 25 \mathrm{~mm})$ and a titanium disk $(\varnothing 12 \times 6 \mathrm{~mm})$ at various contact temperatures. Titanium was studied in three states: CG (initial), UFG (ECAP) and NC $(\mathrm{ECAP}+\mathrm{CR})$. The disk rotation speed was 36 degree $/ \mathrm{s}$. The contact area was heated to temperatures of $150,350,550$, and $800{ }^{\circ} \mathrm{C}$ by passing an electric current, the duration of which was less than $1 \mathrm{~min}$.

A $\varnothing 15 \times 2 \mathrm{~mm}$ disk-shaped composite $\mathrm{Al}_{2} \mathrm{O}_{3} / \mathrm{G}$ was processed by the SPS method of spherical powder of alumina ceramics of various dispersion and five-layer scaly graphene (G) with a content of 0.5 wt.\%. 


\section{Research Results}

\subsection{Copper}

Figure 2 (a) shows the friction coefficient dependence on distance at a load of $5 \mathrm{~N}$ for CG and NC copper. Each curve is characterized by two friction modes. First, the friction coefficient rises sharply, and then reaches the saturation stage. The transition to a stable stage in NC samples takes a longer time than for $\mathrm{CG}$ samples. Although the situation changes the other way around with increasing load up to $10 \mathrm{~N}$, the friction coefficient at the saturation stage for NC copper is always less than for CG copper.

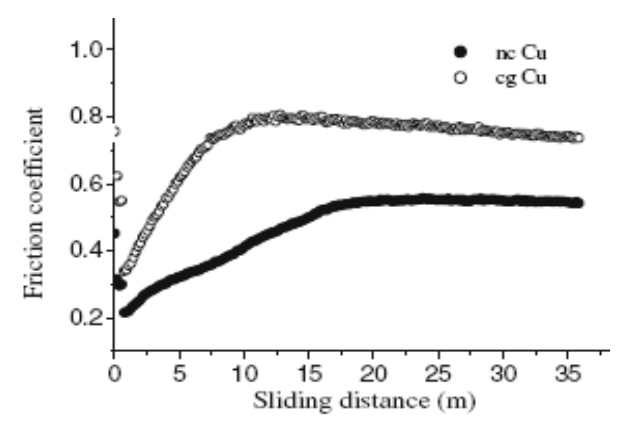

a

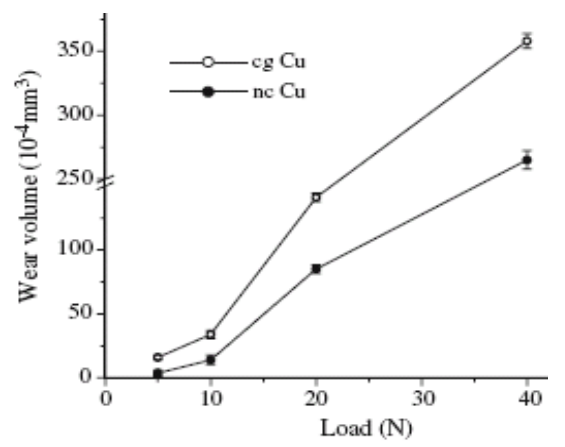

b

Fig. 2. Friction coefficient at a load of $5 \mathrm{~N}$ (a) and wear (b) for CG and NC copper [5].

The change in the value of wear and tear from the load for short circuit and NS copper is shown in Fig. 2 (b). Depreciation increases with increasing load, but the wear rate for NC copper was lower than for CG copper. The microhardness measurement showed that it is twice as high for $\mathrm{NC}$ copper $(1050 \mathrm{MPa})$ as for $\mathrm{CG}$ copper $(500 \mathrm{MPa})$. Thus, a lower friction coefficient and wear in NC copper than for $\mathrm{CG}$ copper is associated with increased microhardness, which in turn is due to the smaller grain size. It is assumed that other reasons may be features related to the refinement of the structure - increased oxidation during friction and weaker strain hardening.

\subsection{Nickel}

Table 1 gives the grain size, microhardness and friction coefficient. The grain size ranged from 8 to $28 \mathrm{~nm}$. The microhardness of $\mathrm{Ni}$ with a grain size of 8 and $10 \mathrm{~nm}$ was close, which is unusual for the general dependence on the grain size It was previously shown that in this size range there is a deviation from the Hall-Petch law, not only quantitatively, but also in sign. This may be due to a change in the deformation mechanism from purely dislocation slip in polycrystalline nickel to intergranular slippage in a nanocrystalline sample.

Table 1. Grain size, microhardness and friction coefficient of electrodeposited nickel.

\begin{tabular}{|c|c|c|}
\hline Grain size, $\mathrm{nm}$ & Microhardness, $\mathrm{MPa}$ & Friction coefficient \\
\hline 61000 & 2690 & 0.62 \\
\hline 28 & 5720 & 0.55 \\
\hline 10 & 7240 & 0.29 \\
\hline 8 & 6890 & 0.16 \\
\hline
\end{tabular}

Figure 3 shows the dependence of the friction coefficient on the number of cycles for various grain sizes. In all cases, this value increases in the first period (up to 500 cycles) of cycling, and then reaches a stable saturation stage (presented in the table). An extremely 
low friction coefficient $(\mathrm{k}=0.16)$ was obtained for nickel with a grain size of $8 \mathrm{~nm}$. In contrast, nickel with the largest grain size of 61 microns showed the maximum value of the coefficient of friction.

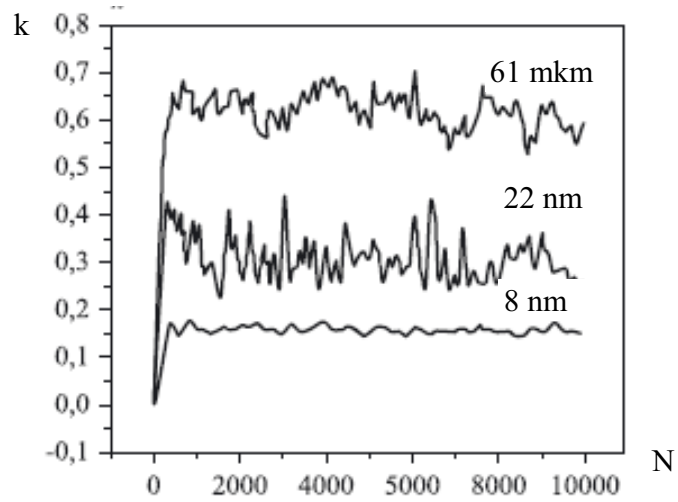

Fig. 3. Friction coefficient dependence for $\mathrm{Ni}$ on the number of cycles and grain sizes [6].

Thus, the study of nanocrystalline nickel showed that the higher microhardness of the electrodeposited sample, associated with the smallest grain size, is the main reason for the decrease in the friction coefficient.

\subsection{Titanium}

Figure 4 shows the temperature dependences $\mu_{\mathrm{a}}$ of titanium in three states at different loads. At all temperatures above room temperature, $C G$ titanium has a higher friction coefficient than UFG or NC titanium. In addition, it increases with temperature for CG titanium and, as a rule, decreases for UFG titanium (except for the state of ECAP + CR at $480 \mathrm{~N}$ and ECAP Ti at $960 \mathrm{~N}$.

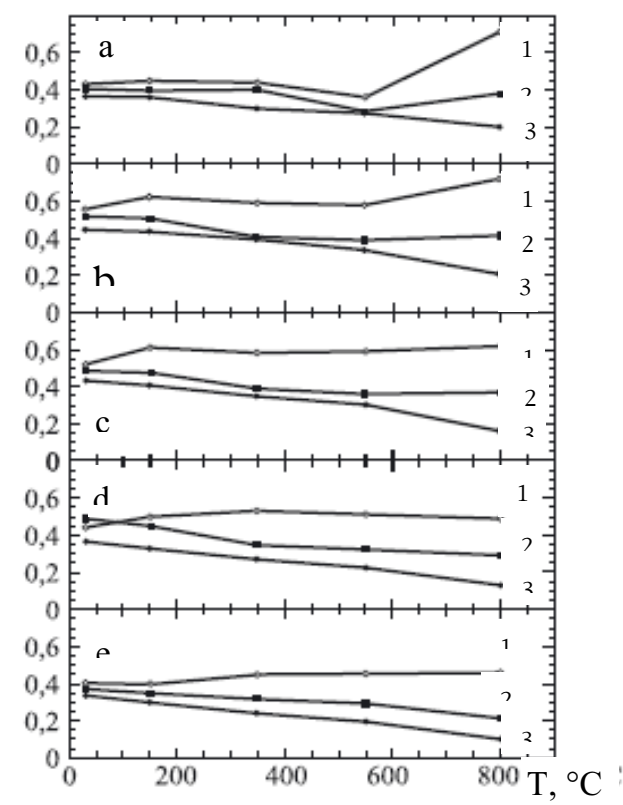

Fig. 4. Temperature dependences of the adhesive component of the friction coefficient in CG (1), UFG (2) and NC (3) titanium at loads: a - $480 \mathrm{H} ; \mathrm{b}-960 \mathrm{H}$; c - $1440 \mathrm{H} ; \mathrm{d}-1920 \mathrm{H}$; e - $2400 \mathrm{H}$ [7]. 
Thus, the formation of UFG or NC structures in titanium reduces the adhesive component of friction $\mu_{\mathrm{a}}$ and the tendency of titanium to stick and set. It is also important that $\mu_{\mathrm{a}}$ decreases with increasing temperature and grain size. As subsequent studies of mechanical behaviour have shown, this effect is associated with multiple hardening of titanium as a result of structure refinement. A similar effect of reducing friction and wear during the structure refinement to the nanometer range was observed in titanium alloys [10].

\subsection{Ceramic composite}

Table 2 shows the microhardness, wear and friction characteristics for the $\mathrm{Al} 2 \mathrm{O} 3 / 0.5 \% \mathrm{G}$ composite. With increasing dispersion of the matrix phase, microhardness naturally increases, and tribological characteristics decrease. This is especially pronounced in the wear characteristics, which are almost halved when the submicron matrix is replaced by a nanocrystalline one.

Table 2. Wear and friction coefficient of $\mathrm{Al}_{2} \mathrm{O}_{3}$ composites.

\begin{tabular}{|c|c|c|c|}
\hline Dispersity & Wear $\left(10^{-3} \mathrm{~mm}^{3}\right)$ & $\begin{array}{c}\text { Wear rate } \mathrm{Kv}\left(10^{-6}\right. \\
\mathrm{mm} / \mathrm{N} \cdot \mathrm{m})\end{array}$ & Friction coefficient \\
\hline submicron & 6.38 & $7.97 \pm 0.05$ & $0.61 \pm 0.05$ \\
\hline nanocrystalline & 3.54 & $4.42 \pm 0.05$ & $0.53 \pm 0.05$ \\
\hline
\end{tabular}

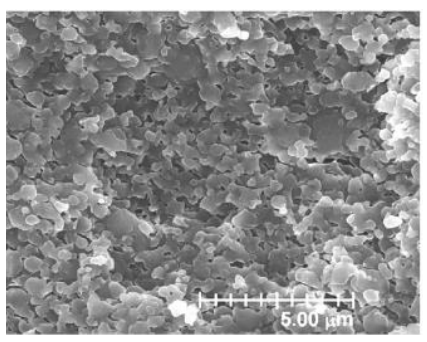

a

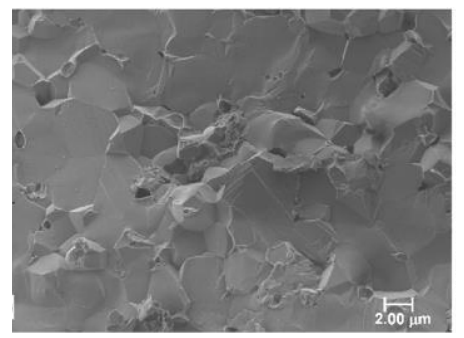

b

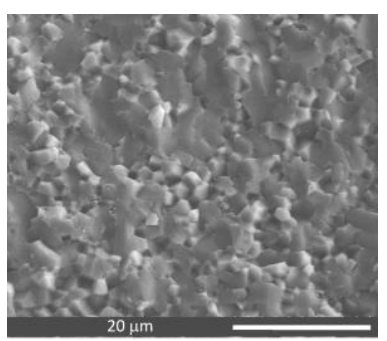

C

Fig. 5. Fracture surface of sintered nanoceramic: a - without graphene; $b, c$ - with graphene $0.5 \%$ (large and low magnification)

An analysis of the fracture surface patterns of nanoceramic (Fig. 5) suggests that the addition of even minimum graphene content can reduce porosity and thereby contribute to an increase in wear resistance.

\subsection{Friction and wear models}

To calculate the adhesion and friction forces acting between the contacting surfaces of solids, methods of continuous contact mechanics based on the linear theory of elasticity are used. The surfaces are assumed to be smooth and even; their atomic structure is not taken into account. For example, the force required to tear off a surface with a radius of curvature $R$ from a flat substrate (Fig. 6) is found by the simple formula $F=3 p R g$, where $p$ is a dimensionless coefficient that takes into account surface roughness and elastic compression at the contact point; $g$ is the surface energy.

This expression can be generalized to other geometries by replacing $\mathrm{R}$ with the corresponding characteristic length. In some cases, the continuum theory gives surprisingly accurate results, and sometimes diverges from experiment tenfold. The reason for this is that the surfaces of real solids in most cases are very different from their idealized mathematical images. Their roughness significantly affects the strength of adhesion. 


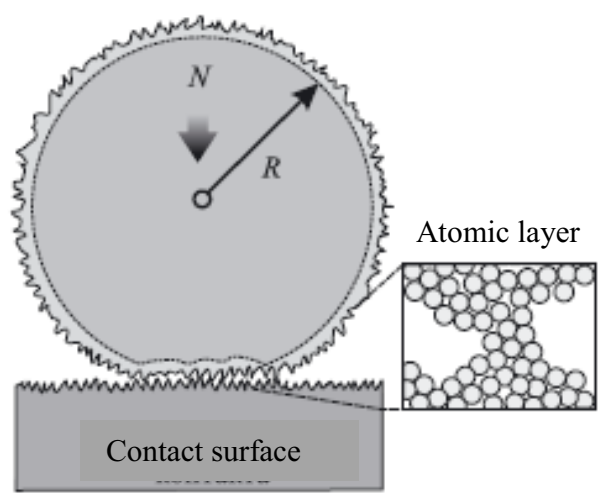

Fig. 6. Mechanical contact of two solids: continual (dashed line) and real surface shape [11].

In addition, there has been a recent surge in interest in micro- and even nanoscale electromechanical devices. They often fail to work due to unwanted adhesion. A continuum models, in principle, are not suitable for calculating their characteristics. All this suggests the need to develop a new, microscopic theory of contact mechanical phenomena.

A step in this direction was taken in [12], the authors of which are from Johns Hopkins Univ. (USA) used the molecular dynamics method to test the limits of applicability of the macroscopic description of contacting surfaces. They studied the contacts between a flat (001) FCC crystal substrate and three different types of cylindrical surfaces having the same radius of curvature (Fig. 7): (a) a curved crystal lattice with an atomically smooth surface, (b) the surface of a sample cut from amorphous solid, and (c) the "stepped" surface of the sample cut from the crystal. In cases (b) and (c), the surface roughness did not exceed one effective atomic diameter (one average interatomic distance). First, the dependences of the displacement $\mathrm{d}$, the contact radius a, and the static friction force $\mathrm{F}$ on the magnitude of the pressing force $\mathrm{N}$ directed along the normal to the substrate were calculated. It turned out that for all types of surfaces the calculated dependences $d(N)$ are in excellent agreement with the predictions of the continuum model. For the dependences a $(\mathrm{N})$, the qualitative agreement between the numerical results and the analytics is preserved, but the quantitative discrepancy reaches $100 \%$.

a

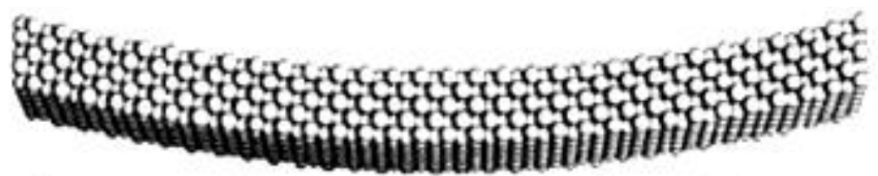

b

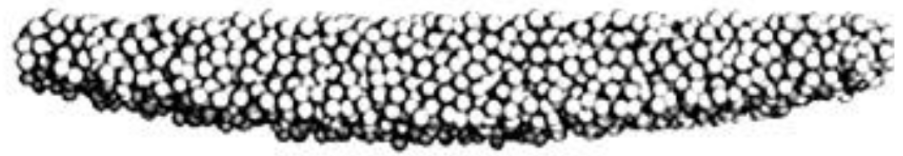

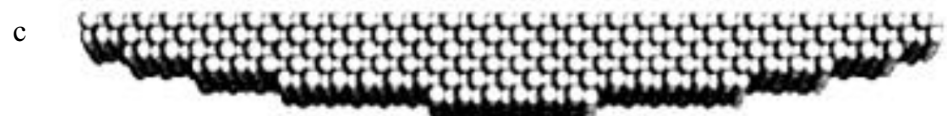

Fig. 7. Surfaces of the same radius but with different structures at the atomic level [8]. 
First of all, this concerns the "stepped" surface, for which a increases with increasing N not "monotonously", but "jumps". The continuum approximation can give a significantly reduced contact area, especially for small N. If for a smooth and "stepped" crystalline surfaces the friction force $\mathrm{F}$ within $(10-20) \%$ coincides with its value expected from macroscopic approach, then for an amorphous sample it turned out to be $\sim 5$ times less. The pressure distribution over the contact area is also very sensitive to the surface structure at the atomic level and in some cases qualitatively differs even with the same roughness, quantified in terms of the standard deviation of the atoms from the ideally smooth surface. Thus, the roughness is an average value that does not allow us to unambiguously predict the mechanical characteristics of the contact without detailing the specific type of atomic disorder, which creates the same roughness.

The results obtained are of both fundamental and practical importance. They not only make it possible to understand the physical nature of contact phenomena better, but also pave the way for "surface engineering", which will make it possible to make contacts with the required macroscopic properties by proper microprocessing of the contacting surfaces.

So far, only the limiting case of small strains has been more or less studied, which corresponds to the contacts of metals or ceramics. In addition, the description of the interaction of "real surfaces" in the "real time" mode requires the mandatory consideration of non-stationary phenomena. These and many other questions that have not yet been resolved is the subject of further theoretical and experimental research.

\section{Conclusion}

The tribological behavior of the pure metals and ceramic composites in the nanocrystalline and traditional coarse-grained states is significantly different. The friction coefficient and the amount of wear in nanocrystalline materials are minimal and are due to the maximum value of microhardness, which in turn depends on the degree of structure refinement. The transition to nanocrystalline contact surfaces can be carried out within the framework of models based on the surface relief at the atomic level.

The work has been supported by Russian Science Foundation [grant № 16-19-10213].

\section{References}

1. Y.T. Zhu, Z. L. Wang, T.G. Langdon, J. Mater Sci 421401(2007)

2. E.N.Hahn, M.A. Mater. Sci.Eng.,A 646 101(2015)

3. S. Hassan, S. Sharma, B. Kumar, Inter. Ref. J. Eng. Sci. 6, 66 (2017)

4. N. Gao, C.T. Wang, R. J. K. Wood, T.G. Langdon, J. Mater. Sci.47 4779 (2012)

5. Z. Han, L. Lu, K. Lu, Tribol. Lett., 21, 47 (2006)

6. B. Basu, R. Balasubramaniam, R. Mishra, Mater. Sci. Eng., A 373370 (2004)

7. V. V. Stolyarov, L. Sh. Shuster, M. Sh. Migranov, R.Z. Valiev, Y.T. Zhu, Mater. Sci. Eng., A 371, 313 (2004)

8. L. Sh. Shuster, Adhesive interaction of a cutting tool with the processed material, M: Mashinostroenie, 96 (1988) in Russian

9. G. S. Fox-Rabinovich, A. J. Kovalev, L. Sh. Shuster, Yu. F. Bokiy, G. K. Dosbayeva, D. L. Wainstein, V. P. Mishina, Wear 206214 (1997)

10. S. Chertovskikh, L. Shuster, V. Stolyarov, Friction and wear, 1, 80 (2005)

11. J. N. Israelachvili, Nature, 435, 893 (2005)

12. B. Luan, M. O. Robbins, Nature, 435, 929 (2005) 